\title{
BRIDGE MONITORING WITH HARMONIC EXCITATION AND PRINCIPAL COMPONENT ANALYSIS
}

\author{
VIET HA NGUYEN ${ }^{1 *}$, JEAN-CLAUDE GOLINVAL ${ }^{2}$, \\ STEFAN MAAS ${ }^{1}$ \\ ${ }^{1}$ University of Luxembourg, Luxembourg, Luxembourg \\ ${ }^{2}$ University of Liege, Liège, Belgium
}

Received 24 November 2017; accepted 23 October 2018

\begin{abstract}
Principal Component Analysis is used for damage detection in structures excited by harmonic forces. Time responses are directly analysed by Singular Value Decomposition to deduct two dominant Proper Orthogonal Values corresponding to two Proper Orthogonal Modes. Damage index is defined by the concept of subspace angle that a subspace is built from the two Proper Orthogonal Modes. A subspace angle reflects the coherence between two different structural health states. An example is given through the application on a part of a real prestressed concrete bridge in Luxembourg where different damage states were created by cutting a number of prestressed tendons in four scenarios with increasing levels. Results are better by using excitation frequency close to an eigenfrequency of the structure. The technique is convenient for practical application in operational bridge structures.
\end{abstract}

Keywords: bridge structure, damage detection, forced harmonic excitation, principal component analysis, subspace angle, time response.

* Corresponding author. E-mail: vietha.nguyen@uni.lu 


\section{Introduction}

Both static and dynamic tests can be carried out to perform the monitoring of bridge structures. Static data are widely used to deduce deflection or inclination of bridges directly. Their measurement is quite fast and often implemented simultaneously with static load testing. Measurements in several positions in a bridge give a direct global vision of the structure (Nguyen, Schommer, Maas, \& Zürbes, 2016). Static load testing means generally charging with important weight, for example, some trucks or lorries/wagons, which are under or sometimes over the design load capacity of the structure. A series of instruments, e.g. transducer, levelling or other devices as laser scan, photogrammetry or GPS exist for measuring structural responses. The last-mentioned devices allow catching the observation of the whole structure easily. These visual devices appear very interesting for large structures with their practical advantages, but their precision plays a substantial role.

Dynamic tests show attractive aspects since they enable monitoring even with a few points of measurement. From vibrational data, dynamic features are identified for structural health monitoring (Peeters \& De Roeck, 20-01). Some dynamic features are usually examined as eigenfrequency, mode shape and damping ratio. These features allow deducing other structural matrices namely stiffness and flexibility under certain conditions (Schommer, Mahowald, Nguyen, Waldmann, Maas, Zürbes, \& De Roeck, 2017), or a variety of detection indexes. There are moreover some methods giving detection indexes directly without any modal identification. For example, based on Principal Component Analysis (PCA) - a multivariable statistical method that observation matrix can be in either time or frequency domain. Combining PCA with Hankel matrices of time data (Yan \& Golinval, 2006), it is possible to characterise the dynamic behaviour of a structure and compare it to a reference state. The comparison is carried out with the help of some subspaces representing dynamic behaviour. In the frequency domain, Nguyen \& Golinval (2010) decomposed Frequency Response Functions (FRFs) and sensitivity analysis in beam-like structures to localise and assess the damage. To deal with temperature effects, Yan, Kerschen, De Boe, \& Golinval (2005) considered a series of frequencies in the observation matrix. The method was proven to be efficient for monitoring some real-life bridges in Nguyen, Mahowald, Golinval, \& Maas (2014).

Kerschen \& Golinval (2002) studied several cases of unforced/forced excitations and revealed that eigenmodes could be assessed by performing PCA on time responses. Some conditions are included: the structure is
Bridge Monitoring with Harmonic

Excitation and Principal Component Analysis 
lightly damped and unforced linear system; the harmonic force with constant amplitude and the mass matrix is proportional to identity as well as the number of samples is large enough. The analysis was performed more extensively for a damped truss structure in the laboratory subjected to harmonic excitation in (Golinval, 2017). The time responses of the structure were measured by both experiments and simulations based on experimentally assessed damping ratios. The present paper aims to exploit the mentioned method for real bridges, out of laboratory conditions. It deals with a part extracted from a real prestressed bridge undergoing several damage states. Sine swept vibrations of low rate excited the structure and interesting issues for detection are obtained.

\section{Principal Component Analysis for responses to harmonic excitation}

\subsection{Principal Component Analysis in brief}

Principal Component Analysis is as a multivariable statistical method and known as efficient for a compressed representation of data both in time and frequency domains, which is widely used for detection problems. A detailed description of the method was given in Kerschen \& Golinval (2002) for general cases, and in Golinval (2017) for the case of harmonic excitation. In the present paper, PCA is briefly presented within the time domain.

Let us consider an observation matrix including the $m$ vibrational signals sampled in $\mathrm{N}$ times $\mathbf{X} \in \mathbf{R}^{m \times N}$ with zero-mean normalisation. Proper orthogonal modes (POMs) and proper orthogonal values (POVs) is be assessed by PCA by performing the Singular Value Decomposition (SVD) on $\mathbf{X}$ :

$$
\mathbf{X}=\mathbf{U S V}^{T}
$$

where $\mathbf{U}$ and $\mathbf{V}$ are $(m \times m)$ and $(N \times N)$ orthogonal matrices respectively; $\mathbf{S}$ is an $(m \times N)$ diagonal matrix containing decreasing singular values. As shown in Kerschen \& Golinval (2002), the columns of $\mathbf{U}$ correspond to POMs of the structure that each POM is associated to a POV, representing by the corresponding singular value in $\mathbf{S}$. This value measures the energy ratio of the associated POM in comparison to the total energy and is defined by the sum of all POVs. The definition of POM is important in the vibrational analysis. Indeed, it is proven that the POMs converge to the eigenmodes in the case of un-damped and unforced linear system, when the mass matrix is proportional to identity and $\mathbf{X}$ contains a sufficient number of samples. 


\subsection{The case of harmonic excitation}

The equation of motion for a damped system submitted to harmonic excitation is often written as below:

$$
\mathbf{M} \ddot{\mathbf{x}}+\mathbf{C} \dot{\mathbf{x}}+\mathbf{K} \mathbf{x}=\mathbf{F} \sin (\omega t)
$$

where $\mathbf{M}, \mathbf{C}$, and $\mathbf{K}$ are the mass, damping and stiffness matrices respectively; $\mathbf{x}$ presents the vector of displacement; $\mathbf{F}$ and $\omega$ are amplitude and the frequency of the excitation.

It is proven by Golinval (2017) in the case of harmonic excitation; there are only two non-zero singular values (POVs). In this case, the forced harmonic responses are captured by only two POMs regardless of the number of measured signals.

$$
\mathbf{X}=\underbrace{\left[\begin{array}{lll}
\frac{\mathscr{R}(\mathbf{H}) \mathbf{F}}{\|\mathcal{R}(\mathbf{H}) \mathbf{F}\|} & \frac{\mathcal{F}(\mathbf{H}) \mathbf{F}}{\|\mathcal{F}(\mathbf{H}) \mathbf{F}\|} & \mathbf{A}
\end{array}\right]}_{\mathbf{U}} \underbrace{\left[\mathbf{I}_{1}\right.}_{\mathbf{S}}] \underbrace{\left[\begin{array}{lll}
\frac{\mathbf{e}_{s}}{\left\|\mathbf{e}_{s}\right\|} & \frac{\mathbf{e}_{c}}{\left\|\mathbf{e}_{c}\right\|} & \mathbf{B}
\end{array}\right]^{T}}_{\mathbf{V}^{T}}
$$

where the diagonal matrix $\mathbf{I}_{1} \in \mathbf{R}^{m \times N}$ contains only two nonzero elements $\|\mathscr{R}(\mathbf{H}) \mathbf{F}\|\left\|\mathbf{e}_{s}\right\|$ and $\|\mathcal{F}(\mathbf{H}) \mathbf{F}\|\left\|\mathbf{e}_{c}\right\|$. $\mathbf{H}$ defines the matrix of FRFs, while $\mathscr{R}($.$) and \mathscr{F}($.) present real and imaginary parts. $\mathbf{e}_{s}=\left[\sin \left(\omega t_{1}\right) \ldots \sin \left(\omega t_{N}\right)\right]^{T}$ and $\mathbf{e}_{c}=\left[\cos \left(\omega t_{1}\right) \ldots \cos \left(\omega t_{N}\right)\right]^{T}$. Matrices $\mathbf{A}$ and $\mathbf{B}$ do not influence equation (3) since they are multiplied by zero elements in $\mathbf{I}_{1}$, but they can be chosen to make $\mathbf{U}$ and $\mathbf{V}$ unitary matrices. By this way, Eq. (3) presents the SVD of the observation matrix $\mathbf{X}$. The first column vectors (POMs) of $\mathbf{U}$, corresponding to the first non-zero elements of $\mathbf{S}$, can represent the dynamic characteristics of the system. Each POM is a combination of all eigenmodes. However, it may tend to the shape of a mode of which the eigenfrequency is close to the frequency of excitation.

\subsection{Damage index based on subspace angle}

A given health structural state of a system can be represented by the active POMs assessed by PCA, including two components when the excitation is harmonic. The two POMs (the first two columns of $\mathbf{U}$ ), considered as active modes and are used to construct active subspaces (which reflect states of the system) at different moments. A change in the dynamic behaviour modifies consequently the state of the system, reflected by the POMs. This change may be estimated using the concept of subspace angle introduced by Golub \& Van Loan (1996). This concept allows quantifying existing spatial coherence between two vibrational data sets.
Bridge Monitoring

with Harmonic

Excitation

and Principal

Component

Analysis 


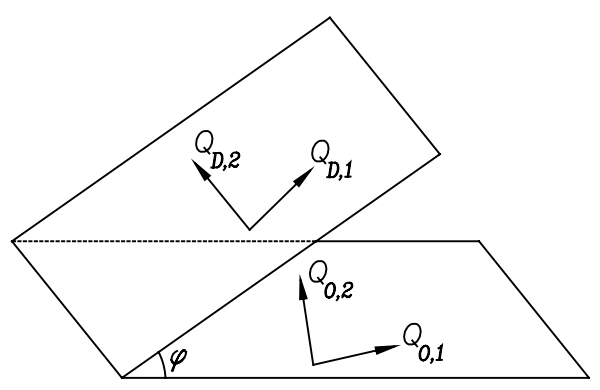

Figure 1. Angle $\varphi$ formed by active subspaces representing the reference and current states

Given two subspaces $\mathbf{O} \in \mathbf{R}^{m \times p}$ and $\mathbf{D} \in \mathbf{R}^{m \times q}$ (each with linear independent columns, $p \geq q$ ), the following $\mathrm{QR}$ factorisations are performed:

$$
\begin{array}{ll}
\mathbf{0}=\mathbf{Q}_{o \mathbf{R}_{0}} & \mathbf{Q}_{O} \in \mathbf{R}^{m \times p} \\
\mathbf{D}=\mathbf{Q}_{D} \mathbf{R}_{D} & \mathbf{Q}_{D} \in \mathbf{R}^{m \times q}
\end{array}
$$

The columns of $\mathbf{Q}_{o}$ and $\mathbf{Q}_{D}$ define the orthonormal bases for $\mathbf{O}$ and $\mathbf{D}$ respectively. The two subspaces $\mathbf{O}$ and $\mathbf{D}$ formed between them an angle $\varphi_{\mathrm{i}}$ that can be computed from singular values associated with the product $\mathbf{Q}_{O}^{T} \mathbf{Q}_{D}$ :

$$
\mathbf{Q}_{O}^{T} \mathbf{Q}_{D}=\mathbf{U}_{O D} \Sigma_{O D} \mathbf{V}_{O D}^{T} ; \Sigma_{O D}=\operatorname{diag}\left(\cos \varphi_{i}\right), i=1, \ldots, q
$$

The largest singular value is thus related to the largest angle characterising the geometric difference between two subspaces. An example is given in Figure 1 for 2-dimensional active subspaces (hyperplanes), following a dynamic change.

\section{Application}

\subsection{Harmonic exciters and the testing structure}

In University of Luxembourg (UL), the research on bridge monitoring has been carried out since more than one decade. In real circumstances, the complexity of the monitoring is envisaged, e.g. different conditions of temperature, bearing or foundation situation, excitation level. The research team in the UL used forced harmonic exciters while implementing dynamic tests for bridge structures in Luxembourg for the sake of controlling excitation forces. Two exciters were built and used for different ranges of frequency and amplitude forces to assure an equivalent excitation for different tests (Maas, Zürbes, Waldmann, Waltering, 


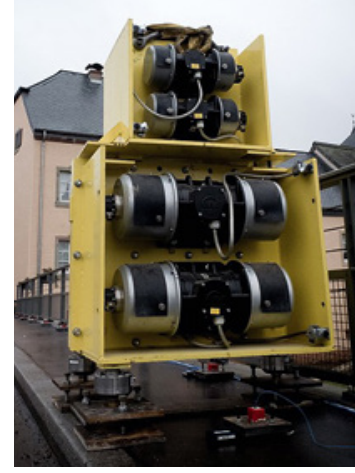

a) eccentric mass exciter

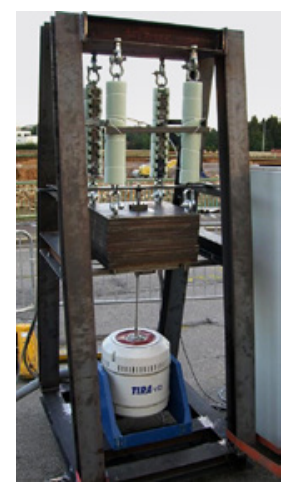

b) electromagnetic exciter

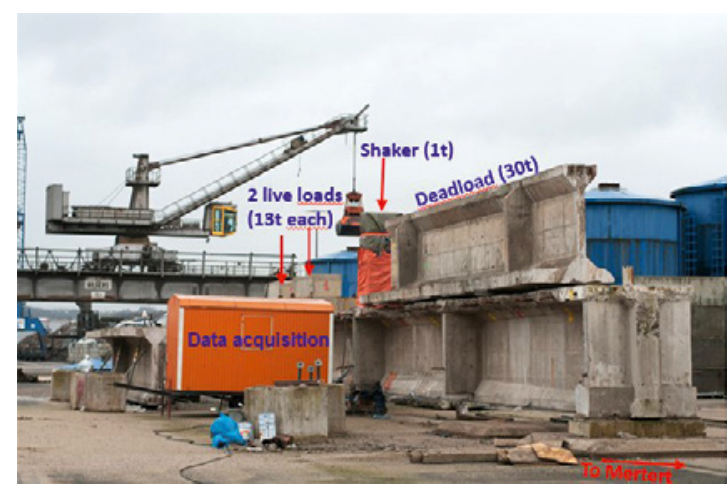

c) test set-up

Figure 2. Forced harmonic exciters and the test set-up

Bungard, \& De Roeck, 2012). It consists in an eccentric mass exciter (force amplitudes above $10 \mathrm{kN}$ for $f>4 \mathrm{~Hz}$ ) and an electromagnetic exciter with a feedback control loop (force amplitudes up to $2.7 \mathrm{kN}$ ) presented in Figures $2 \mathrm{a}$ and $2 \mathrm{~b}$. The advantage while keeping the same amplitude of excitation is the level of responses is equivalent, which makes easier the comparison among different damage states. It is feasible to complete modal testing for a bridge in one day by two persons with a real measurement time of a few minutes. The transport is possible with a small lorry, and the system is installed at the site on the sidewalk. When everything is ready, the traffic is stopped during a few minutes to execute the measurements. This key feature enables minimising measurement noise, and the dependency on the excitation force is excluded. Moreover, only short-time measurements are needed, as a clear generation of measurable and adjustable harmonic swept sine excitation are introduced as input forces into the structure.

The structure to test is a part of a real prestressed concrete bridge built in Luxembourg during the 1950s and demolished in 2013. Therefore, the UL had an opportunity to perform a series of tests in

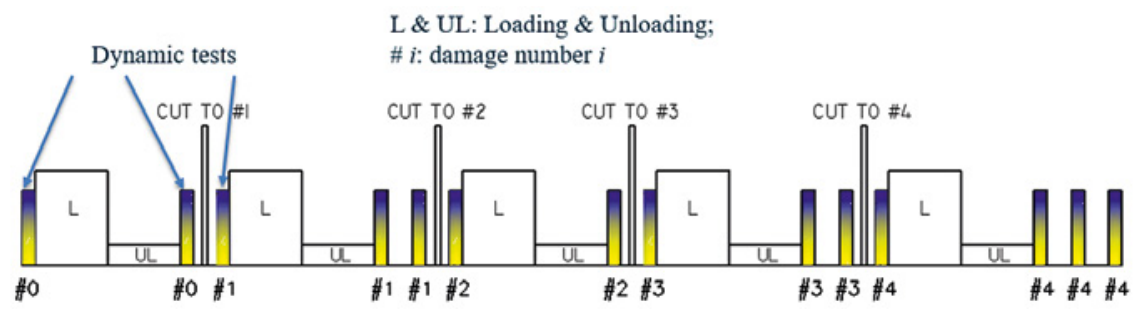

Figure 3. Process of tests 


\begin{tabular}{cccccc}
\hline Scenario \# & $\mathbf{0}$ & $\mathbf{1}$ & $\mathbf{2}$ & $\mathbf{3}$ & $\mathbf{4}$ \\
\hline Number of tendons cut & 0 & 2 & 4 & 6 & $\approx 9$ \\
\hline
\end{tabular}

a piece of this bridge, as shown in Figure 2c. Good results of static loading tests were reported recently in Nguyen, Schommer, Maas, \& Zürbes (2016) and Schommer, Nguyen, Maas, \& Zürbes (2017). The current paper presents some results accordingly from the dynamic test, performed by the shaker shown in Figure $2 \mathrm{~b}$.

Both static and dynamic tests were realised throughout the initial and damaged states of the bridge. Damages were created by cutting a number of prestressed tendons. The Table 1 describes the examined damage scenarios by the cutting from the 19 prestressed tendons. Horizontal cracks appeared from the first damage scenario and vertical cracks only from the third scenario.

Vibrational tests were performed with swept sine excitations with the amplitude of $2000 \mathrm{~N}$ and frequency varied from $2.5 \mathrm{~Hz}$ to $25 \mathrm{~Hz}$ with a weak rate of $0.02 \mathrm{~Hz} / \mathrm{s}$. Responses were sampled at an interval $\Delta t=0.0004 \mathrm{~s}$. It implies that a time response of 2500 samplings may relate to unchanged excitation frequency or a maximum difference of $0.02 \mathrm{~Hz}$. Referring to Eq. (2), such a period can be considered as constant excitation frequency and so as harmonic excitation. Its number of samples is still large enough for the statistical requirement of PCA. Moreover, since the amplitude of the excitation was kept constant, a zero-mean normalisation for the data is no longer necessary. Principal Component Analysis is performed based on 11-time responses recorded along the longitudinal axis of the structure.

\subsection{Results}

All different health conditions from the initial situation \#0 to the most serious damage \#4 are processed and laid together. For analysis, all states are examined by periods of which the frequency of excitation comes close a structural eigenfrequency. Figure 4 displays the POVs of every state while considering time signals according to excitation frequency nearby the first structural eigenfrequency of about $3 \mathrm{~Hz}$. The SVD was carried out for the observation matrix. It is shown that the first POD holds the most important energy as the corresponding POV keeps more than $90 \%$ of the total 11 POVs. Values decrease typically from the third POV, which means only two POVs are significant. The remaining POVs, despite their minor non-zero values, are supposed as noisy existing in real measurements and insignificant. 


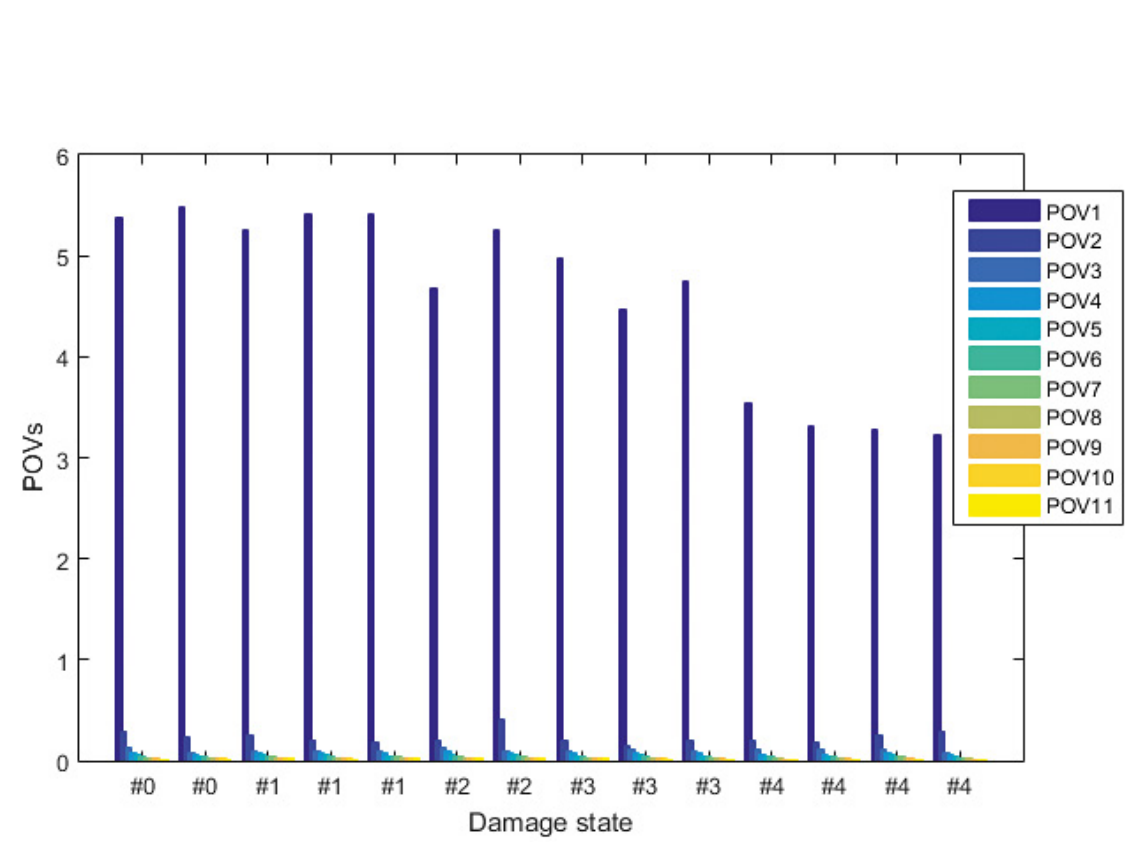

Viet Ha Nguyen,

Jean-Claude

Golinval,

Stefan Maas

Bridge Monitoring

with Harmonic

Excitation

and Principal

Component

Analysis

Figure 4. Proper orthogonal values (POV) of all damage states

Figure 4 reveals clearly that the first POV is reduced when the level of damage increases. An overall view shows three grades including similar values: the initial state \#0 and the lowest level \#1, damages \#2 and \#3 while all tests \#4 have the smallest POVs. This grading may relate to the monotonous reduction of the first Eigen frequency as shown in Figure 5 .

With periods of which the excitation frequency is nearby higher eigenfrequency, the evolution of the POVs does not reveal a clear trend relating to the levels of damage. Correspondingly, the reduction of higher eigenfrequency is smaller and much less monotonous.

$\Delta f_{1}$

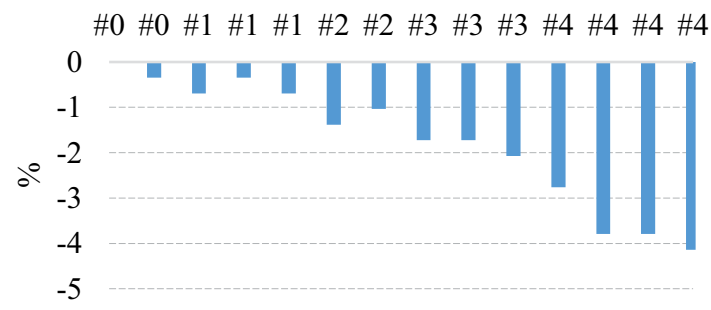

Figure 5. Changes of the first eigenfrequencies $\Delta f_{1}$ identified throughout the whole test 

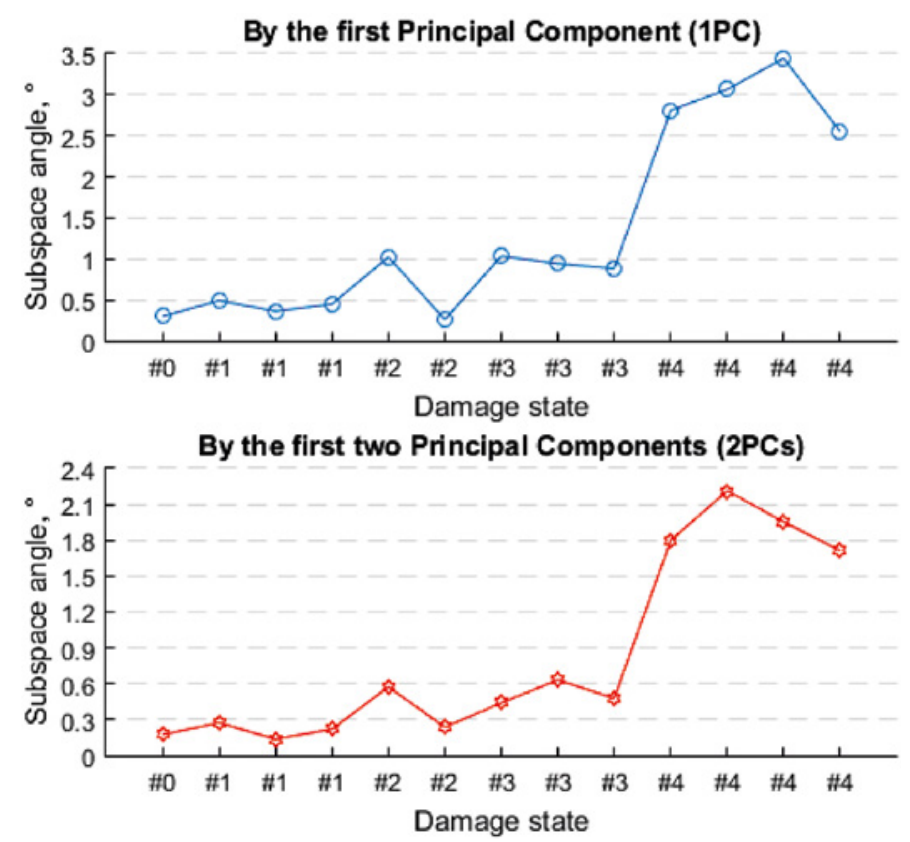

Figure 6 . Damage indexes by subspace angles, the frequency of excitation is nearby $f_{1}$
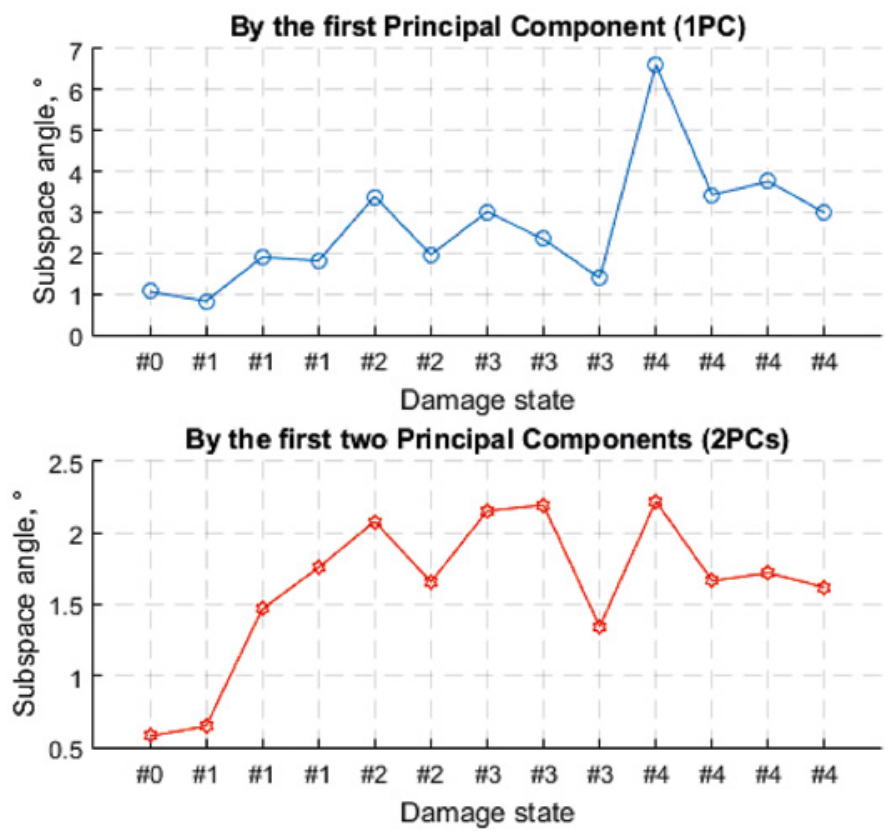

Figure 7. Damage indexes by subspace angles, the frequency of excitation is nearby $f_{2}$ 
By taking into account the first two POMs for each health state, it Bridge Monitoring with Harmonic is possibly characterised by a subspace of two vectors. The concept of subspace angle allows quantifying the coherence of different states.

Subspace angles are presented in Figures 6 and 7 when considering excitation frequencies near the first and second eigenfrequencies respectively. These detections are better than other periods.

The examination of Figure 6, while the excitation frequency being nearby the first eigenfrequency, and both two images give quite a good detection. Based on subspaces built from only the first POM or two POMs, damages are noticed from damage state \#3 and damage \#4 has very distinct indexes. On the other hand, Figure 7 presents the case when the excitation frequency is around the second resonance. Based on only the first POM or two POMs, the last damage \#4 does not correspond to highest indexes, but damage can be detected from \#1. The first test of \#1 is not distinguished from the initial state \#0; maybe this test was performed just after the cutting of two tendons, before the loading of the static test. The two following tests of \#1 give larger indexes due to the crack extension after the static loading, which was finished before these two vibrational tests.

\section{Conclusions}

1. The paper deals with the detection problem in considering vibrational responses from swept sine excitations with low sweep rate in a real ridge structure. Although the testing was carried out in a part extracted from an intact bridge, a similar test procedure can be realised in an operational bridge. In operational condition, it is possible to stop the traffic for a few minutes because vibrational measurements last less than a half hour.

2. Since noise always exists, there are more than two non-zero proper orthogonal values. However, the first two proper orthogonal values are the most dominant. It is proven when the excitation frequency is nearby an eigenfrequency, the first proper orthogonal value often occupies more than $95 \%$ of the total energy, and the damage detection is more efficient. 


\section{REFERENCES}

Golinval, J. C. (2017). Damage detection in structures based on principal component analysis of forced harmonic responses. Procedia engineering, 199, 1912-1918. https://doi.org/10.1016/j.proeng.2017.09.449

Golub, G. H., \& Van Loan, C. F. (1996). Matrix computations. 1996. Johns Hopkins University, Press, Baltimore, MD, USA, 374-426.

Kerschen, G., \& Golinval, J. C. (2002). Physical interpretation of the proper orthogonal modes using the singular value decomposition. Journal of Sound and vibration, 249(5), 849-865. https://doi.org/10.1006/jsvi.2001.3930

Maas, S., Zürbes, A., Waldmann, D., Waltering, M., Bungard, V., \& De Roeck, G. (2012). Damage assessment of concrete structures through dynamic testing methods. Part 2: Bridge tests. Engineering Structures, 34, 483-494. https://doi.org/10.1016/j.engstruct.2011.09.018

Hà, N. V., \& Golinval, J. C. (2010). Localization and quantification of damage in beam-like structures using sensitivities of principal component analysis results. Mechanical Systems and Signal Processing, 24(6), 1831-1843. https://doi.org/10.1016/j.ymssp.2010.01.012

Nguyen, V. H., Mahowald, J., Golinval, J. C., \& Maas, S. (2014). Damage detection in civil engineering structure considering temperature effect. In Dynamics of Civil Structures, Volume 4 (pp. 187-196). Springer, Cham. https://doi.org/10.1007/978-3-319-04546-7_22.

Nguyen, V. H., Schommer, S., Maas, S., \& Zürbes, A. (2016). Static load testing with temperature compensation for structural health monitoring of bridges. Engineering Structures, 127, 700-718. https://doi.org/10.1016/j.engstruct.2016.09.018

Peeters, B., \& De Roeck, G. (2001). Stochastic system identification for operational modal analysis: a review. Journal of Dynamic Systems, Measurement, and Control, 123(4), 659-667. https://doi.org/10.1115/1.1410370

Schommer, S., Mahowald, J., Nguyen, V. H., Waldmann, D., Maas, S., Zürbes, A., \& De Roeck, G. (2017). Health Monitoring based on Dynamic Flexibility matrix: Theoretical Models versus in-situ Tests. Engineering, 9(02), 37-67. https://doi.org/10.4236/eng.2017.92004

Schommer, S., Nguyen, V. H., Maas, S., \& Zürbes, A. (2017). Model updating for structural health monitoring using static and dynamic measurements. Procedia engineering, 199, 2146-2153. https://doi.org/10.1016/j.proeng.2017.09.156

Yan, A. M., Kerschen, G., De Boe, P., \& Golinval, J. C. (2005). Structural damage diagnosis under varying environmental conditions-part I: a linear analysis. Mechanical Systems and Signal Processing, 19(4), 847-864. https://doi.org/10.1016/j.ymssp.2004.12.002

Yan, A. M., \& Golinval, J. C. (2006). Null subspace-based damage detection of structures using vibration measurements. Mechanical Systems and Signal Processing, 20(3), 611-626. https://doi.org/10.1016/j.ymssp.2005.04.010 